\title{
TWO-DIMENSIONAL PULSE PROPAGATION IN A TWO-PARAMETER ANISOTROPIC ELASTIC SOLID*
}

\author{
ROBERT G. PAYTON \\ Adelphi University
}

Summary. A perturbing body force, in the form of an impulsive point source, is suddenly introduced into an anisotropic elastic body. The anisotropic solid is characterized by two parameters. After placing suitable restrictions on these parameters, integral representations of the transient displacement components are found. Explicit expressions for the displacements are obtained along two perpendicular lines which are centered at the point of application of the point impulse and parallel to the coordinate axes. Wave front singularities are identified and graphical results for the wave shapes are presented.

1. Introduction. In this paper a study is made of the linearized, two-dimensional, impulsive motion of an anisotropic elastic solid. The solid is characterized by (essentially) two free parameters (stretch parameters) and could, for example, model a finitely strained body excited by a small perturbing body force.

In order to insure explicit results herein, certain restrictions are placed on the stretch parameters (see Sec. 3, below). Even so, the displacements have only been found along two perpendicular lines (centered on the point impulse body force) and parallel to the $x$ and $y$ axes. In Sec. 5 the wave front singularities are briefly discussed and numerical results are presented for the displacement components, in Figs. 3 and 4, when the stretch parameters take on specific values.

As typical of other recent work on anisotropic elastic wave propagation, the papers of Scott and Miklowitz [1] and Cameron and Eason [2] may be mentioned.

2. The equations of motion. The problem to be investigated herein concerns the plane motion of an anisotropic elastic solid whose displacements $u(x, y, t)$ and $v(x, y, t)$ are governed by the equations

and

$$
L_{11}[u]+L_{12}[v]=-\rho_{0} a_{1} \delta\left(x-x_{0}\right) \delta\left(y-y_{0}\right) \delta(t),
$$

$$
L_{21}[u]+L_{22}[v]=-\rho_{0} a_{2} \delta\left(x-x_{0}\right) \delta\left(y-y_{0}\right) \delta(t) \text {. }
$$

The differential operators $L_{i j}$ are given by

$$
\begin{aligned}
& L_{11}=2 \lambda_{1}^{2} a_{11} \frac{\partial^{2}}{\partial x^{2}}+2 \lambda_{2}^{2} a_{12} \frac{\partial^{2}}{\partial y^{2}}-\rho_{0} \frac{\partial^{2}}{\partial t^{2}}, \\
& L_{22}=2 \lambda_{1}^{2} a_{21} \frac{\partial^{2}}{\partial x^{2}}+2 \lambda_{2}^{2} a_{22} \frac{\partial^{2}}{\partial y^{2}}-\rho_{0} \frac{\partial^{2}}{\partial t^{2}}
\end{aligned}
$$

and

$$
L_{12}=L_{21}=2 \mu \lambda_{1}^{2} \lambda_{2}^{2} \frac{\partial^{2}}{\partial x \partial y}
$$

* Received November 7, 1967; revised version received March 18, 1968. Research sponsored by the Air Force Office of Scientific Research, Office of Aerospace Research, United States Air Force under AFOSR Grant No. 68-1442. 
The constants $a_{i j}$ are related to the positive numbers $\lambda_{1}$ and $\lambda_{2}$ (stretch parameters) by

$$
\begin{aligned}
& a_{11} / \mu=9 \lambda_{1}^{2} / 4+\lambda_{2}^{2} / 4-1, \\
& a_{22} / \mu=\lambda_{1}^{2} / 4+9 \lambda_{2}^{2} / 4-1
\end{aligned}
$$

and

$$
a_{21} / \mu=a_{12} / \mu=3\left(\lambda_{1}^{2}+\lambda_{2}^{2}\right) / 4-1 .
$$

Here $u$ is the (horizontal) displacement component parallel to the $x$-axis and $v$ is the (vertical) displacement component parallel to the $y$-axis. The mass density of the body is denoted by $\rho_{0}$ and $\mu$ is a physical constant having the dimensions of force per unit area. The forcing terms on the right-hand side of Eqs. (2.1) correspond to a body force in the form of a point impulse of strength $\left(a_{1}, a_{2}\right)$ in the $(x, y)$ direction.

Since the constants $\mu$ and $\rho_{0}$ can be absorbed into the time variable, there are basically two free parameters in the problem, $\lambda_{1}$ and $\lambda_{2}$. In this connection it is worth noting that in the special case $\lambda_{1}=\lambda_{2}=1$, Eqs. (2.1) reduce to the two-dimensional Navier equations for an isotropic elastic solid, provided both Lamé parameters are set equal to $\mu$.

3. Integral transform set up for the plane strain problem. By means of the linear transformation

$$
x^{\prime}=x-x_{0} \text { and } y^{\prime}=y-y_{0},
$$

the origin in the $\left(x^{\prime}, y^{\prime}\right)$ plane is made to coincide with the location of the source. The equations of motion (2.1) then become

$$
L_{11}^{\prime}\left[u^{\prime}\right]+L_{12}^{\prime}\left[v^{\prime}\right]=-\rho_{0} a_{1} \delta\left(x^{\prime}\right) \delta\left(y^{\prime}\right) \delta(t),
$$

and

$$
L_{21}^{\prime}\left[u^{\prime}\right]+L_{22}^{\prime}\left[v^{\prime}\right]=-\rho_{0} a_{2} \delta\left(x^{\prime}\right) \delta\left(y^{\prime}\right) \delta(t),
$$

where the primed operators are the same as those in (2.2) after replacing $x$ and $y$ by $x^{\prime}$ and $y^{\prime}$. Also

and

$$
u(x, y, t)=u\left(x^{\prime}+x_{0}, y^{\prime}+y_{0}, t\right) \equiv u^{\prime}\left(x^{\prime}, y^{\prime}, t\right)
$$

$$
v(x, y, t)=v\left(x^{\prime}+x_{0}, y^{\prime}+y_{0}, t\right) \equiv v^{\prime}\left(x^{\prime}, y^{\prime}, t\right) .
$$

Introduce now the Laplace (in $t$ ) and double Fourier (in $x^{\prime}$ and $y^{\prime}$ ) transforms by

$$
\tilde{\tilde{u}}^{* \prime}\left(\xi_{1}, \xi_{2}, s\right)=\int_{0}^{\infty} \int_{-\infty}^{+\infty} \int_{-\infty}^{+\infty} u^{\prime}\left(x^{\prime}, y^{\prime}, t\right) \exp \left(-s t-i \xi_{1} x^{\prime}-i \xi_{2} y^{\prime}\right) d x^{\prime} d y^{\prime} d t,
$$

and inverse transforms by

$u^{\prime}\left(x^{\prime}, y^{\prime}, t\right)=\frac{1}{(2 \pi)^{3} i} \int_{\Gamma} \int_{-\infty}^{+\infty} \int_{-\infty}^{+\infty} \tilde{\bar{u}}^{* \prime}\left(\xi_{1}, \xi_{2}, s\right) \exp \left(s t+i \xi_{1} x^{\prime}+i \xi_{2} y^{\prime}\right) d \xi_{1} d \xi_{2} d s$.

A bar will be used to denote the Laplace transform (with parameter $s$ ) and a tilde and star to denote the Fourier transforms (with parameters $\xi_{1}$ and $\xi_{2}$ ). Assuming now that the body occupies the entire $\left(x^{\prime}, y^{\prime}\right)$ plane and that initially (i.e. prior to the application of the point source) the body is at rest, the transformed equations (3.2) become

$$
\left[2 \lambda_{1}^{2} a_{11} \xi_{1}^{2}+2 \lambda_{2}^{2} a_{12} \xi_{2}^{2}+\rho_{0} s^{2}\right] \tilde{u}^{* \prime}+\left[2 \mu \lambda_{1}^{2} \lambda_{2}^{2} \xi_{1} \xi_{2}\right] \tilde{\tilde{v}}^{* \prime}=\rho_{0} a_{1}
$$


and

$$
\left[2 \mu \lambda_{1}^{2} \lambda_{2}^{2} \xi_{1} \xi_{2}\right] \tilde{\bar{u}}^{* \prime}+\left[2 \lambda_{1}^{2} a_{21} \xi_{1}^{2}+2 \lambda_{2}^{2} a_{22} \xi_{2}^{2}+\rho_{0} s^{2}\right] \tilde{v}^{* \prime}=\rho_{0} a_{2}
$$

The system determinant of the two algebraic equations (3.5) is given by

$$
\begin{gathered}
\Delta=4 \lambda_{1}^{4} a_{11} a_{21} \xi_{1}^{4}+\xi_{1}^{2}\left[4 \lambda_{1}^{2} \lambda_{2}^{2} a_{11} a_{22} \xi_{2}^{2}+2 \lambda_{1}^{2} a_{11} \rho_{0} s^{2}+4 \lambda_{1}^{2} \lambda_{2}^{2} a_{12}^{2} \xi_{2}^{2}+2 \lambda_{1}^{2} a_{12} \rho_{0} s^{2}-4 \mu^{2} \lambda_{1}^{4} \lambda_{2}^{4} \xi_{2}^{2}\right] \\
+\left[2 \lambda_{2}^{2} a_{12} \xi_{2}^{2} \rho_{0} s^{2}+2 \lambda_{2}^{2} a_{22} \rho_{0} s^{2} \xi_{2}^{2}+\rho_{0}^{2} s^{4}+4 \lambda_{2}^{4} a_{12} a_{22} \xi_{2}^{4}\right] .
\end{gathered}
$$

The following assumption will now be made

(i) The roots of $\Delta=0$, regarded as a function of $\xi_{1}^{2}$, are to be negative and distinct.

Condition (i) will be shown below to place certain restrictions on the stretch parameters $\lambda_{1}$ and $\lambda_{2}$. So far nothing has been said about the type of the system of partial differential equations (3.2). However, the number of initial conditions used on (3.2) in going to (3.5) has tacitly assumed that the system (3.2) is totally hyperbolic. A sufficient condition for the system (3.2) to be totally hyperbolic is, according to Courant and Hilbert [3], that the $s$ roots of $\Delta=0$ are pure imaginary and distinct. Condition (i) above is somewhat more restrictive than that needed for hyperbolicity and is used here for mathematical convenience. Condition (i) will force certain branch point singularities [see Sec. 4 below] to lie on the imaginary axis, as they do in the case of isotropic elasticity theory for the corresponding problem.

The equation

$$
\Delta=0
$$

is a quadratic equation in $\xi_{1}^{2}$. After dividing through by $a_{11} a_{21}$, the condition that both of these roots be negative certainly requires that the coefficients of $\xi_{1}^{2}$ and $\xi_{1}^{0}$ be positive. Furthermore, if this is to hold uniformly in $\xi_{2}^{2}$, then $\left(1 / a_{12}+1 / a_{11}\right)>0$ (from the coefficient of $\xi_{1}^{2}$ as $\xi_{2}^{2} \rightarrow 0$ ), $a_{22} / a_{11}>0$ (from the coefficient of $\xi_{1}^{0}$ as $\xi_{2}^{2} \rightarrow \infty$ ), and $1 /\left(a_{11} a_{12}\right)>0$ (from the coefficient of $\xi_{1}^{0}$ as $\xi_{2}^{2} \rightarrow 0$ ). Here $s$, the Laplace transform parameter, has been treated as a positive constant. It is easily seen (by contradiction) that the above three inequalities are satisfied if

$$
a_{11}>0, \quad a_{12}=a_{21}>0 \text { and } a_{22}>0 .
$$

It is now advantageous to change from the $\xi_{1}, \xi_{2}$ transform parameters to $\zeta_{1}, \zeta_{2}$ by means of the substitutions

$$
\lambda_{1} \xi_{1}=\zeta_{1} s\left(\frac{\rho_{0}}{2 a_{12}}\right)^{1 / 2} \text { and } \lambda_{2} \xi_{2}=\zeta_{2} s\left(\frac{\rho_{0}}{2 a_{12}}\right)^{1 / 2} .
$$

Then

$$
\begin{aligned}
\frac{\Delta}{\rho_{0}^{2} s^{4}}=\frac{a_{11}}{a_{12}} \zeta_{1}^{4}+\zeta_{1}^{2} \zeta_{2}^{2}\left(\frac{a_{11} a_{22}}{a_{12}^{2}}+1-\frac{\lambda_{1}^{2} \lambda_{2}^{2} \mu^{2}}{a_{12}^{2}}\right) & +\frac{a_{22}}{a_{12}} \zeta_{2}^{4} \\
& +\zeta_{1}^{2}\left(\frac{a_{11}}{a_{12}}+1\right)+\zeta_{2}^{2}\left(\frac{a_{22}}{a_{12}}+1\right)+1 .
\end{aligned}
$$

If

$$
a_{11} / a_{12}=\alpha, \quad a_{22} / a_{12}=\beta
$$

then from $(2.3)$

$$
\mu^{2} \lambda_{1}^{2} \lambda_{2}^{2} / a_{12}^{2}=(3 \alpha+\beta)(\alpha+3 \beta) / 16-(\alpha+\beta)+1
$$


so that

$$
\Delta / \rho_{0}^{2} s^{4}=\alpha \zeta_{1}^{4}+A \zeta_{1}^{2}+B
$$

where

$$
A=\gamma \zeta_{2}^{2}+(\alpha+1), \quad B=\left(\beta \zeta_{2}^{2}+1\right)\left(\zeta_{2}^{2}+1\right)
$$

and

$$
\gamma=-3(\alpha-\beta)^{2} / 16+(\alpha+\beta) .
$$

In terms of the new parameters $\alpha$ and $\beta$

$$
\begin{gathered}
\lambda_{1}^{2}=\frac{3 \alpha+\beta-4}{3 \alpha+3 \beta-10}, \quad \lambda_{2}^{2}=\frac{\alpha+3 \beta-4}{3 \alpha+3 \beta-10} \\
\frac{a_{11}}{\mu}=\frac{4 \alpha}{3 \alpha+3 \beta-10}, \quad \frac{a_{12}}{\mu}=\frac{4}{3 \alpha+3 \beta-10} \quad \text { and } \quad \frac{a_{22}}{\mu}=\frac{4 \beta}{3 \alpha+3 \beta-10}
\end{gathered}
$$

Because of conditions (3.8) and the fact that $\lambda_{1}^{2}$ and $\lambda_{2}^{2}$ are necessarily positive, attention is restricted to that portion of the $(\alpha, \beta)$ plane for which

$\alpha>0, \quad \beta>0, \quad 3(\alpha+\beta)-10>0, \quad 3 \alpha+\beta-4>0, \quad$ and $\alpha+3 \beta-4>0$.

The five conditions of Eq. (3.15), while necessary, are not sufficient to insure that the $\xi_{1}^{2}$ roots of (3.7) are negative and distinct.

From Eq. (3.12) it is seen that in order for the $\xi_{1}^{2}$ roots of (3.7) to be negative and distinct

$$
A>0, B>0 \text { and }\left(A^{2}-4 \alpha B\right)>0,
$$

these conditions holding uniformly for $0 \leq \zeta_{2}^{2}<\infty$. Since $\beta>0$, Eq. (3.13) shows that $B>0$ is automatically satisfied. Thus if there are any further restrictions on the allowable $\alpha, \beta$ values they must come from the conditions $A>0$ and $\left(A^{2}-4 \alpha B\right)>0$. Also by Eq. (3.13) certainly $A>0$ if

$$
\gamma>0
$$

Now

$$
A^{2}-4 \alpha B=\zeta_{2}^{4}\left(\gamma^{2}-4 \alpha \beta\right)+2 \zeta_{2}^{2}[(\alpha+1) \gamma-2 \alpha(\beta+1)]+(\alpha-1)^{2},
$$

so that $\left(A^{2}-4 \alpha B\right)$ will be positive if

$$
\text { Case (1) } \quad\left(\gamma^{2}-4 \alpha \beta\right)>0 \text { and }(\alpha+1) \gamma-2 \alpha(\beta+1)>0, \quad(\alpha \neq 1)
$$

or if

$$
\text { Case (2) } \quad \begin{gathered}
\left(\gamma^{2}-4 \alpha \beta\right)>0, \quad(\alpha+1) \gamma-2 \alpha(\beta+1)<0, \quad(\alpha \neq 1) \quad \text { and } \\
(\alpha-1)^{2}\left(\gamma^{2}-4 \alpha \beta\right)-[(\alpha+1) \gamma-2 \alpha(\beta+1)]^{2}>0 .
\end{gathered}
$$

But the third inequality in Eq. (3.20) can be simplified to

$$
(\alpha-\beta)^{2}(\alpha+3 \beta-4)(3 \alpha+\beta-4)<0,
$$

which, in view of Eqs. (3.15), cannot be satisfied. Therefore, case (2) is ruled out. Finally, 
the inequalities of case (1) will be satisfied when

$$
\gamma>2(\alpha \beta)^{1 / 2}>2 \alpha(\beta+1) /(\alpha+1),
$$

these conditions being compatible with a portion of the $(\alpha, \beta)$ plane meeting conditions (3.15).

In summary the allowable $(\alpha, \beta)$ region $R$ (see Fig. 1 ) is the triangular region defined by

$$
\begin{aligned}
\alpha & =0, & & 4 \leq \beta \leq 16 / 3 \\
3 \alpha+\beta & =4, & & 3 \leq \beta \leq 4
\end{aligned}
$$

and

$$
\beta=\left(\alpha^{1 / 2}+4 / 3^{1 / 2}\right)^{2}, \quad 3 \leq \beta \leq 16 / 3 .
$$

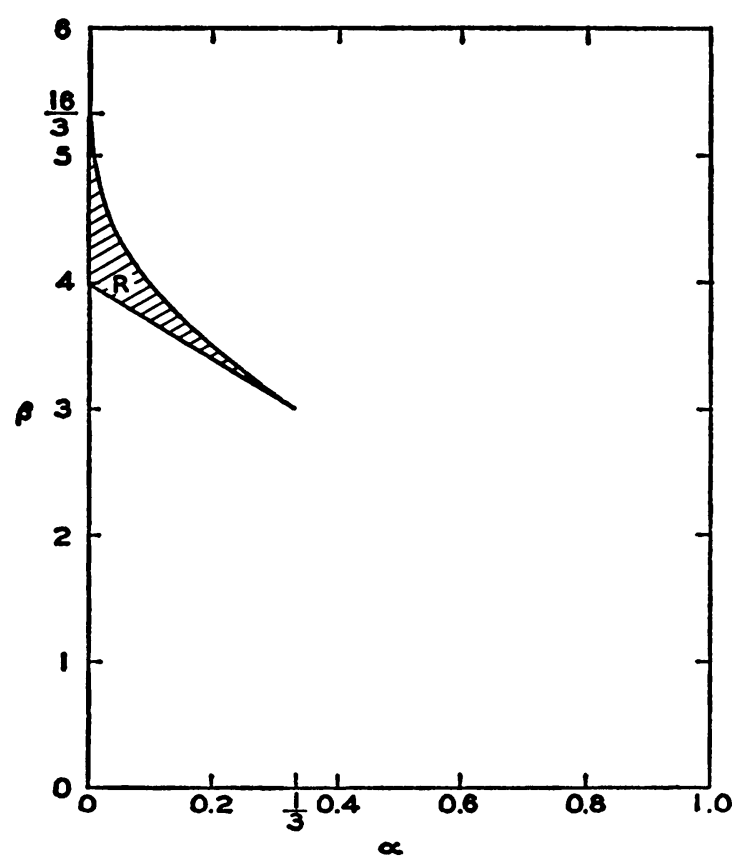

Fig. 1. Allowable $\alpha, \beta$ region $R$

Then if $(\alpha, \beta)$ lies in the interior of $R$ (not on the boundary) the inequalities (3.15) and (3.21) are satisfied, with the consequence that the $\zeta_{1}^{2}$ roots of (3.7) are distinct and negative. As pointed out above, restricting $(\alpha, \beta)$ to $R$ is stronger than the hyperbolicity requirement. In fact the region $R$ does not include the simple case $\alpha=\beta$ wherein $\Delta$ is easily factored as in the corresponding case for isotropic elasticity.

4. Inversion of the displacement transforms. If the algebraic equations (3.5) are solved for the transformed displacements and the Fourier inversion formulae of (3.4) are invoked (taking account of Eqs. (3.9)) then

$$
\begin{aligned}
\bar{u}^{\prime}\left(x^{\prime}, y^{\prime}, s\right)=\frac{\rho_{0}}{2 \mu \alpha \lambda_{1}^{2} \lambda_{2}^{2}} \frac{1}{(2 \pi)^{2}} \int_{-\infty}^{+\infty} \int_{-\infty}^{+\infty}\left[\frac{a_{1} K^{1 / 2}\left(\zeta_{1}^{2}+\beta \zeta_{2}^{2}+1\right)-a_{2} K\left(\zeta_{1} \zeta_{2}\right)}{\left(\zeta_{1}^{2}+\phi_{1}^{2}\right)\left(\zeta_{1}^{2}+\phi_{2}^{2}\right)}\right] \\
\cdot \exp \left(i m s\left(\zeta_{1} x^{\prime} / \lambda_{1}+\zeta_{2} y^{\prime} / \lambda_{2}\right)\right) d \zeta_{1} d \zeta_{2},
\end{aligned}
$$


and

$$
\begin{aligned}
\bar{v}\left(x^{\prime}, y^{\prime}, s\right)=\frac{\rho_{0}}{2 \mu \alpha \lambda_{1}^{2} \lambda_{2}^{2}} \frac{1}{(2 \pi)^{2}} \int_{-\infty}^{+\infty} \int_{-\infty}^{+\infty}\left[\frac{a_{2} K^{1 / 2}\left(\alpha \zeta_{1}^{2}+\zeta_{2}^{2}+1\right)-a_{1} K\left(\zeta_{1} \zeta_{2}\right)}{\left(\zeta_{1}^{2}+\phi_{1}^{2}\right)\left(\zeta_{1}^{2}+\phi_{2}^{2}\right)}\right] \\
\cdot \exp \left(i m s\left(\zeta_{1} x^{\prime} / \lambda_{1}+\zeta_{2} y^{\prime} / \lambda_{2}\right)\right) d \zeta_{1} d \zeta_{2}
\end{aligned}
$$

where

$$
\begin{aligned}
\phi\left(\zeta_{2}\right) & =\left[\frac{A-\left(A^{2}-4 \alpha B\right)^{1 / 2}}{2 \alpha}\right]^{1 / 2}, \quad \phi_{2}\left(\zeta_{2}\right)=\left[\frac{A+\left(A^{2}-4 \alpha B\right)^{1 / 2}}{2 \alpha}\right]^{1 / 2}, \\
K & =(\alpha+3 \beta-4)(3 \alpha+\beta-4) / 16 \text { and } m=\left(\rho_{n} / 2 a_{12}\right)^{1 / 2} .
\end{aligned}
$$

Note that, because of the restrictions placed on $\alpha$ and $\beta, \phi_{1}\left(\zeta_{2}\right)$ and $\phi_{2}\left(\zeta_{2}\right)$ are positive throughout the entire $\zeta_{2}$ integration range.

Since the integrands of Eqs. (4.1) and (4.2), regarded as a function of $\zeta_{1}$, have as their only singularities simple poles at $\pm i \phi_{1}$ and $\pm i \phi_{2}$, the $\zeta_{1}$ integration is easily performed. Accounting for the odd or even properties of the subsequent $\zeta_{2}$ integrands gives

$$
\begin{aligned}
\bar{u}^{\prime}\left(x^{\prime}, y^{\prime}, s\right)=\frac{\rho_{0} a_{1} K^{1 / 2}}{4 \mu \alpha \lambda_{1}^{2} \lambda_{2}^{2}}\left[\bar{I}_{1}\left(x^{\prime}, y^{\prime}, s\right)\right. & \left.-\bar{I}_{2}\left(x^{\prime}, y^{\prime}, s\right)\right] \\
& +\frac{\rho_{0} a_{2} K}{4 \mu \alpha \lambda_{1}^{2} \lambda_{2}^{2}} \frac{y^{\prime}}{\left|y^{\prime}\right|}\left[\bar{I}_{3}\left(x^{\prime}, y^{\prime}, s\right)-\bar{I}_{4}\left(x^{\prime}, y^{\prime}, s\right)\right],
\end{aligned}
$$

and

$$
\begin{aligned}
\bar{v}^{\prime}\left(x^{\prime}, y^{\prime}, s\right)=\frac{\rho_{0} a_{2} K^{1 / 2}}{4 \mu \alpha \lambda_{1}^{2} \lambda_{2}^{2}}\left[\bar{I}_{5}\left(x^{\prime}, y^{\prime}, s\right)\right. & \left.-\bar{I}_{6}\left(x^{\prime}, y^{\prime}, s\right)\right] \\
& +\frac{\rho_{0} a_{1} K}{4 \mu \alpha \lambda_{1}^{2} \lambda_{2}^{2}} \frac{y^{\prime}}{\left|y^{\prime}\right|}\left[\bar{I}_{3}\left(x^{\prime}, y^{\prime}, s\right)-\bar{I}_{4}\left(x^{\prime}, y^{\prime}, s\right)\right],
\end{aligned}
$$

where

$$
\begin{aligned}
& \bar{I}_{1}=\operatorname{Re}_{\pi} \frac{1}{\pi} \int_{0}^{\infty} \frac{-\phi_{1}+\beta \zeta_{2}+1}{\phi_{1}\left(\phi_{2}^{2}-\phi_{1}^{2}\right)} \exp \left[\operatorname{sm}\left(-\left|x^{\prime}\right| \phi_{1} / \lambda_{1}+i\left|y^{\prime}\right| \zeta_{2} / \lambda_{2}\right)\right] d \zeta_{2}, \\
& \bar{I}_{2}=\operatorname{Re} \frac{1}{\pi} \int_{0}^{\infty} \frac{-\phi_{2}^{2}+\beta \zeta_{2}^{2}+1}{\phi_{2}\left(\phi_{2}^{2}-\phi_{1}^{2}\right)} \exp \left[\operatorname{sm}\left(-\left|x^{\prime}\right| \phi_{2} / \lambda_{1}+i\left|y^{\prime}\right| \zeta_{2} / \lambda_{2}\right)\right] d \zeta_{2}, \\
& \bar{I}_{3}=\operatorname{Im} \frac{1}{\pi} \int_{0}^{\infty} \frac{\zeta_{2}}{\phi_{2}^{2}-\phi_{1}^{2}} \exp \left[\operatorname{sm}\left(-\left|x^{\prime}\right| \phi_{1} / \lambda_{1}+i\left|y^{\prime}\right| \zeta_{2} / \lambda_{2}\right)\right] d \zeta_{2}, \\
& \bar{I}_{4}=\operatorname{Im} \frac{1}{\pi} \int_{0}^{\infty} \frac{\zeta_{2}}{\phi_{2}^{2}-\phi_{1}^{2}} \exp \left[\operatorname{sm}\left(-\left|x^{\prime}\right| \phi_{2} / \lambda_{1}+i\left|y^{\prime}\right| \zeta_{2} / \lambda_{2}\right)\right] d \zeta_{2}, \\
& \bar{I}_{5}=\operatorname{Re} \frac{1}{\pi} \int_{0}^{\infty} \frac{-\alpha \phi_{1}^{2}+\zeta_{2}^{2}+1}{\phi_{1}\left(\phi_{2}^{2}-\phi_{1}^{2}\right)} \exp \left[\operatorname{sm}\left(-\left|x^{\prime}\right| \phi_{1} / \lambda_{1}+i\left|y^{\prime}\right| \zeta_{2} / \lambda_{2}\right)\right] d \zeta_{2},
\end{aligned}
$$

and

$$
\bar{I}_{6}=\operatorname{Re} \frac{1}{\pi} \int_{0}^{\infty} \frac{-\alpha \phi_{2}^{2}+\zeta_{2}^{2}+1}{\phi_{2}\left(\phi_{2}^{2}-\phi_{1}^{2}\right)} \exp \left[\operatorname{sm}\left(-\left|x^{\prime}\right| \phi_{2} / \lambda_{1}+i\left|y^{\prime}\right| \zeta_{2} / \lambda_{2}\right)\right] d \zeta_{2} .
$$

The method of Cagniard [4] will now be used to determine explicit expressions for the $u$ and $v$ displacements along the lines $x=x_{0}\left(x^{\prime}=0\right)$ and $y=y_{0}\left(y^{\prime}=0\right)$. It might 
appear, since no boundaries are involved in the problem treated here, that it should be just as easy to determine the displacements along any line through the origin in the $\left(x^{\prime}, y^{\prime}\right)$ plane. However, the use of the Cagniard method for general $\left(x^{\prime}, y^{\prime}\right)$ values requires a knowledge of the path in the complex $\zeta_{2}$-plane along which the exponent in Eqs. (4.1) and (4.2) is real. Due to the complicated nature of $\phi_{1}\left(\zeta_{2}\right)$ and $\phi_{2}\left(\zeta_{2}\right)$, this path is not easily found. A technique whereby the proper integration path is found numerically has been used by Kraut [j] on a problem involving anisotropic elastic wave motion. In order to keep the $(\alpha, \beta)$ parameters as free as possible (at least along the line $y=y_{0}$ ), such a scheme as Kraut's will not be considered in this paper. Lighthill [6] has also suggested a method for the approximate inversion of integrals such as (4.1) and (4.2). In Lighthill's method the $\left(x^{\prime}, y^{\prime}\right)$ axes are rotated so that one of the axes coincides with the line along which the displacement solutions are desired. The $\left(\zeta_{1}, \zeta_{2}\right)$ axes must also be suitably rotated. It is this latter rotation which spoils the analysis insofar as ease of evaluation goes, since the denominator $\Delta$ in the integrand of Eqs. (4.1) and (4.2) will no longer be a simple quadratic in (the new) $\zeta_{1}^{2}$, but rather a full fourth degree polynominal. Other ways for simplifying such multiple integrals have been presented by John [7] and Gelfand and Shilov [8].

Proceeding now with the displacement evaluations along $y^{\prime}=0$, Eq. (4.4) gives

$$
\bar{u}^{\prime}\left(x^{\prime}, 0, s\right)=\frac{\rho_{0} a_{1} K^{1 / 2}}{4 \mu \alpha \lambda_{1}^{2} \bar{\lambda}_{2}^{2}}\left[\bar{I}_{1}\left(x^{\prime}, 0, s\right)-\bar{I}_{2}\left(x^{\prime}, 0, s\right)\right] .
$$

Now from Eq. (4.6)

$$
\bar{I}_{1}\left(x^{\prime}, 0, s\right)=\frac{1}{\pi} \int_{0}^{\infty} g_{1}\left(\zeta_{2}\right) \exp \left(-s m\left|x^{\prime}\right| \phi_{1} / \lambda_{1}\right) d \zeta_{2},
$$

where

$$
g_{1}\left(\zeta_{2}\right)=-\left(\phi_{1}^{2}+\beta \zeta_{2}^{2}+1\right) / \phi_{1}\left(\phi_{2}^{2}-\phi_{1}^{2}\right) .
$$

The Laplace inversion of (4.13) is now immediate

$$
I_{1}\left(x^{\prime}, 0, t\right)=\frac{1}{\pi} \int_{0}^{\infty} g_{1}\left(\zeta_{2}\right) \delta\left[t-m\left|x^{\prime}\right| \phi_{1} / \lambda_{1}\right] d \zeta_{2},
$$

where $\delta$ is the Dirac delta function. A similar expression can be obtained for $I_{2}\left(x^{\prime}, 0, t\right)$. Calling

$$
\tau=\lambda_{1} t / m\left|x^{\prime}\right|
$$

allows $u^{\prime}\left(x^{\prime}, 0, t\right)=u\left(x^{\prime}+x_{0}, 0, t\right)=u\left(x, y_{0}, t\right)$ to be written as

$$
\frac{16 \pi \mu}{a_{1} \rho_{0}} t u\left(x, y_{0}, t\right)=\frac{(3 \alpha+3 \beta-10)^{2} \tau}{((3 \alpha+\beta-4)(\alpha+3 \beta-4))^{1 / 2}}\left[\frac{h_{1}(\tau)-h_{2}(\tau)}{\alpha}\right],
$$

where

$h_{1}(\tau)=\int_{0}^{\infty} g_{1}\left(\zeta_{2}\right) \delta\left[\tau-\phi_{1}\left(\zeta_{2}\right)\right] d \zeta_{2} \quad$ and $\quad h_{2}(\tau)=\int_{0}^{\infty} g_{2}\left(\zeta_{2}\right) \delta\left[\tau-\phi_{2}\left(\zeta_{2}\right)\right] d \zeta_{2}$,

with $g_{2}\left(\zeta_{2}\right)=\left(-\phi_{2}^{2}+\beta \zeta_{2}^{2}+1\right) / \phi_{2}\left(\phi_{2}^{2}-\phi_{1}^{2}\right)$. In going from (4.12) to (4.16), the relations (3.14) have been used to express the product $\lambda_{1}^{2} \lambda_{2}^{2}$ in terms of $(\alpha, \beta)$. From the sifting property of the delta functions, $h_{1}(\tau)$ will have the form 


$$
h_{1}(\tau)=g_{1}\left(\kappa_{1}\right) /\left|\phi_{1}^{\prime}\left(\kappa_{1}\right)\right|,
$$

where $\kappa_{1}(\tau)$ is the positive root of $\tau-\phi_{1}\left(\zeta_{2}\right)=0$. If there is more than one such root, then $h_{1}(\tau)$ must be summed over all the roots. Because of the restrictions placed on the stretch parameters (see Sec. 3), the function $\psi\left(\zeta_{2}\right)=\tau-\phi_{1}\left(\zeta_{2}\right)$ is a monotonically decreasing function of $\zeta_{2}$ in the range $0 \leq \zeta_{2}<\infty$. Thus in order for $\psi\left(\zeta_{2}\right)=0$ to have a root, $\tau-\phi_{1}(0)$ must be nonnegative. In this case there will be just one root $\kappa_{1}(\tau)$ satisfying $\psi\left(\kappa_{1}\right)=0$. Hence, in order to bring out the wave propagation features, the expression for $h_{1}(\tau)$ in Eq. (4.18) must be multiplied by the Heaviside unit step function $H\left[\tau-\phi_{1}(0)\right]$, thus:

$$
h_{1}(\tau)=\frac{g_{1}\left(\kappa_{1}\right)}{\left|\phi_{1}^{\prime}(\kappa)\right|} H\left[\tau-\phi_{1}(0)\right] .
$$

Omitting the algebra, the complete expression for $h_{1}(\tau)$ is now given by

$$
h_{1}(\tau)=\frac{\alpha\left(\beta \kappa_{1}^{2}+1-\tau^{2}\right) H(\tau-1)}{\kappa_{1}\left(\left[\gamma \tau^{2}-(\beta+1)\right]^{2}-4 \beta\left(\alpha \tau^{2}-1\right)\left(\tau^{2}-1\right)\right)^{1 / \overline{2}}}
$$

where

$\kappa_{1}(\tau)=\left[\frac{\gamma \tau^{2}-(\beta+1)+\left(\left[\gamma \tau^{2}-(\beta+1)\right]^{2}-4 \beta\left(\alpha \tau^{2}-1\right)\left(\tau^{2}-1\right)\right)^{1 / 2}}{2 \beta}\right]^{1 / 2}$.

The expression for $h_{2}(\tau)$ is similarly obtained:

$$
h_{2}(\tau)=\frac{\alpha\left(\beta \kappa_{2}^{2}+1-\tau^{2}\right) H\left(\tau-\alpha^{-1 / 2}\right)}{\kappa_{2}\left(\left[\gamma \tau^{2}-(\beta+1)\right]^{2}-4 \beta\left(\alpha \tau^{2}-1\right)\left(\tau^{2}-1\right)\right)^{1 / 2}},
$$

with

$\boldsymbol{k}_{2}(\tau)=\left[\frac{\gamma \tau^{2}-(\beta+1)-\left(\left[\gamma \tau^{2}-(\beta+1)\right]^{2}-4 \beta\left(\alpha \tau^{2}-1\right)\left(\tau^{2}-1\right)\right)^{1 / 2}}{2 \beta}\right]^{1 / 2}$.

Equations (4.20)-(4.23), when introduced into (4.16), give an explicit expression for the horizontal displacement component along the line $y=y_{0}$ passing through the origin of the impulsive source.

The $v$ displacement along $y=y_{0}$ can be found in exactly the same way as $u\left(x, y_{0}, t\right)$ was determined; hence only the result will be recorded here:

$$
\frac{16 \pi \mu}{a_{2} \rho_{0}} t v\left(x, y_{0}, t\right)=\frac{(3 \alpha+3 \beta-10)^{2} \tau}{((3 \alpha+\beta-4)(\alpha+3 \beta-4))^{1 / 2}}\left[\frac{h_{5}(\tau)-h_{6}(\tau)}{\alpha}\right],
$$

where

$$
h_{5}(\tau)=\frac{\alpha\left(\kappa_{1}^{2}+1-\alpha \tau^{2}\right) H(\tau-1)}{\kappa_{1}\left(\left[\gamma \tau^{2}-(\beta+1)\right]^{2}-4 \beta\left(\alpha \tau^{2}-1\right)\left(\tau^{2}-1\right)\right)^{1 / 2}},
$$

and

$$
h_{6}(\tau)=\frac{\alpha\left(\kappa_{2}^{2}+1-\alpha \tau^{2}\right) H\left(\tau-\alpha^{-1 / 2}\right)}{\kappa_{2}\left(\left[\gamma \tau^{2}-(\beta+1)\right]^{2}-4 \beta\left(\alpha \tau^{2}-1\right)\left(\tau^{2}-1\right)\right)^{1 / 2}} .
$$

In order to evaluate the displacement integrals along the line $x^{\prime}=0$, it is desirable to alter the integration path for the integrals (4.6)-(4.11) so as to coincide with the positive $\operatorname{Im} \zeta_{2}$-axis. In this case (since $x^{\prime}=0$ ) the exponents in these integrals will be real. 
From Eq. (4.4)

$$
\bar{u}^{\prime}\left(0, y^{\prime}, s\right)=\frac{\rho_{0} a_{1} K^{1 / 2}}{4 \mu \alpha \lambda_{1}^{2} \lambda_{2}^{2}}\left[\bar{I}_{1}\left(0, y^{\prime}, s\right)-\bar{I}_{2}\left(0, y^{\prime}, s\right)\right]
$$

where

$$
\bar{I}_{1}\left(0, y^{\prime}, s\right)-\bar{I}_{2}\left(0, y^{\prime}, s\right)=\operatorname{Re} \frac{1}{\pi} \int_{0}^{\infty} F\left(\zeta_{2}\right) \exp \left(m s i\left|y^{\prime}\right| \zeta_{2} / \lambda_{2}\right) d \zeta_{2}
$$

and

$$
F\left(\zeta_{2}\right)=(2 \alpha)^{1 / 2} \frac{1+(\alpha \beta)^{1 / 2}\left[\left(\zeta_{2}^{2}+1 / \beta\right) /\left(\zeta_{2}^{2}+1\right)\right]^{1 / 2}}{\left[A+\left(A^{2}-4 \alpha B\right)^{1 / 2}\right]^{1 / 2}+\left[A-\left(A^{2}-4 \alpha B\right)^{1 / 2}\right]^{1 / 2}} .
$$

Regarded as a function of the complex variable $\zeta_{2}$, the only singularities of $F\left(\zeta_{2}\right)$ are branch points at $\pm i \beta^{-1 / 2}$ and $\pm i$. At the points $\zeta_{2}= \pm i a, \pm i b$ where

$a=\left[\frac{\gamma(\alpha+1)-2 \alpha(\beta+1)+\left([\gamma(\alpha+1)-2 \alpha(\beta+1)]^{2}-(1-\alpha)^{2}\left[\gamma^{2}-4 \alpha \beta\right]\right)^{1 / 2}}{\gamma^{2}-4 \alpha \beta}\right]^{1 / 2}$,

and

$b=\left[\frac{\gamma(\alpha+1)-2 \alpha(\beta+1)-\left([\gamma(\alpha+1)-2 \alpha(\beta+1)]^{2}-(1-\alpha)^{2}\left[\gamma^{2}-4 \alpha \beta\right]\right)^{1 / 2}}{\gamma^{2}-4 \alpha \beta}\right]^{1 / 2}$,

the quantity $\left(A^{2}-4 \alpha B\right)$ vanishes. However, these points are only apparent branch point singularities of $F\left(\zeta_{2}\right)$ since $F\left(\zeta_{2}\right)$ is easily seen to be an even function of $\left(A^{2}-\right.$ $4 \alpha B)^{1 / 2}$. The denominator of $F\left(\zeta_{2}\right)$ does have branch points where $B=0$ but from Eq. (3.13) these are just the points $\pm i \beta^{-1 / 2}$ and $\pm i$. Let the branch cuts for $F\left(\zeta_{2}\right)$ be in the second and third quadrants of the complex $\zeta_{2}$-plane along the half lines $\operatorname{Im} \zeta_{2}= \pm 1$, $\operatorname{Im} \zeta_{2}= \pm \beta^{-1 / 2}$, with $\operatorname{Re} \zeta_{2}<0$. The branches of the various multi-valued expressions of $F\left(\zeta_{2}\right)$ are chosen so that these expressions are positive along the positive $\zeta_{2}$-axis.

If the positive $\zeta_{2}$-axis integration path of (4.28) is joined by a large quarter circle $\left|\zeta_{2}\right| \rightarrow+\infty, 0<\arg \zeta_{2}<\pi / 2$, plus a path returning to the origin along the positive $\operatorname{Im} \zeta_{2}$-axis (see Fig. 2), then

$$
\begin{aligned}
\bar{I}_{1}\left(0, y^{\prime}, s\right)-\bar{I}_{2}\left(0, y^{\prime}, s\right)=\operatorname{Re} & \frac{i}{\pi}\left[\int_{0}^{\beta^{-1 / 2}} F\left(\xi e^{i \pi / 2}\right) \exp \left(-m s\left|y^{\prime}\right| \xi / \lambda_{2}\right) d \xi\right. \\
& +\int_{\beta^{-1 / 2}}^{1} F\left(\xi e^{i \pi / 2}\right) \exp \left(-m s\left|y^{\prime}\right| \xi / \lambda_{2}\right) d \xi \\
& \left.+\int_{1}^{\infty} F\left(\xi e^{i \pi / 2}\right) \exp \left(-m s\left|y^{\prime}\right| \xi / \lambda_{2}\right) d \xi\right]
\end{aligned}
$$

The result of Eq. (4.32) follows from an application of Cauchy's theorem, and the fact that the quarter circle contribution to the contour integral vanishes as $\left|\zeta_{2}\right| \rightarrow \infty$ (by Jordan's lemma) since $F\left(\zeta_{2}\right)=0\left(\left|\zeta_{2}\right|^{-1}\right)$. Furthermore, the real part of the first integral in Eq. (4.32) contributes nothing since $\operatorname{Im} F\left(\xi e^{i \pi / 2}\right)=0$ for $0<\xi<\beta^{-1 / 2}$. Taking the Laplace inverse transform of (4.32) gives 


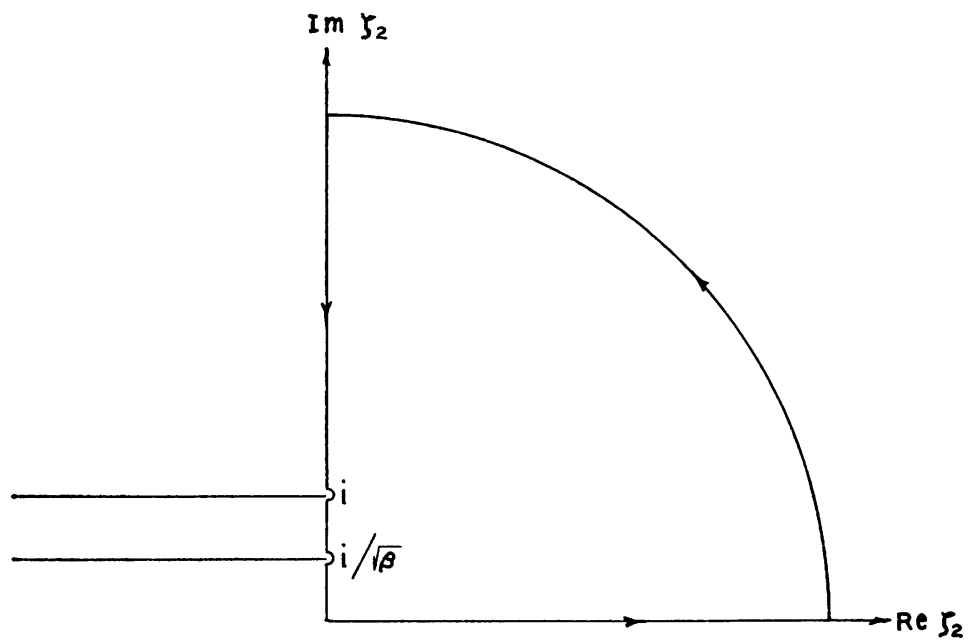

FIG. 2. Branch cuts and integration contour in the complex $\zeta_{2}-$ plane

$$
\begin{aligned}
I_{1}\left(0, y^{\prime}, t\right)-I_{2}\left(0, y^{\prime}, t\right)=\operatorname{Re} \frac{i}{\pi}\left[\int_{\beta^{-1 / 2}}^{1} F\left(\xi e^{i \pi / 2}\right) \delta\left(t-m\left|y^{\prime}\right| \xi / \lambda_{2}\right) d \xi\right. \\
\\
\left.\quad+\int_{1}^{\infty} F\left(\xi e^{i \pi / 2}\right) \delta\left(t-m\left|y^{\prime}\right| \xi / \lambda_{2}\right) d \xi\right] .
\end{aligned}
$$

Calling

$$
\omega=\lambda_{2} t / m\left|y^{\prime}\right|,
$$

allows $u\left(x_{0}, y, t\right)$ to be written as

$$
\frac{16 \pi \mu}{a_{1} \rho_{0}} t u\left(x_{0}, y, t\right)=-\frac{(3 \alpha+3 \beta-10)^{2}}{\alpha((3 \alpha+\beta-4)(\alpha+3 \beta-4))^{1 / 2}}\left[\omega f_{1}(\omega)+\omega f_{2}(\omega)\right],
$$

where

$$
f_{1}(\omega)=\operatorname{Im} F\left(\omega e^{i \pi / 2}\right) H(\omega-1)
$$

and

$$
f_{2}(\omega)=\operatorname{Im} F\left(\omega e^{i \pi / 2}\right)\left[H\left(\omega-\beta^{-1 / 2}\right)-H(\omega-1)\right] .
$$

Explicit expressions for $f_{1}(\omega)$ and $f_{2}(\omega)$ are

$$
\begin{gathered}
\beta^{-1 / 2} \leq \omega<1, \quad f_{2}(\omega)=\frac{(2 \alpha)^{1 / 2}}{A+D+|A+D|}\left[(\alpha \beta)^{1 / 2}(A+D)^{1 / 2}\right. \\
\left.\cdot\left(\frac{\omega^{2}-1 / \beta}{1-\omega^{2}}\right)^{1 / 2}-(|A-D|)^{1 / 2}\right], \\
1<\omega \leq b, \quad f_{1}(\omega)=-(2 \alpha)^{1 / 2} \frac{1+(\alpha \beta)^{1 / 2}\left(\frac{\omega^{2}-1 / \beta}{\omega^{2}-1}\right)^{1 / 2}}{(|A+D|)^{1 / 2}+(|A+D|)^{1 / 2}}, \\
b \leq \omega \leq a, \quad f_{1}(\omega)=-(\alpha)^{1 / 2} \frac{1+(\alpha \beta)^{1 / 2}\left(\frac{\omega^{2}-1 / \beta}{\omega^{2}-1}\right)^{1 / 2}}{\left(|A|+(4 \alpha \beta)^{1 / 2}\right)^{1 / 2}},
\end{gathered}
$$


and

$$
a \leq \omega<\infty, \quad f_{1}(\omega)=-(2 \alpha)^{1 / 2} \frac{1+(\alpha \beta)^{1 / 2}\left(\frac{\omega^{2}-1 / \beta}{\omega^{2}-1}\right)^{1 / 2}}{(|A+D|)^{1 / 2}+(|A-D|)^{1 / 2}},
$$

where $D=\left(A^{2}-4 \alpha B\right)^{1 / 2}$ and the functions $A$ and $B$ (see Eqs. (3.13)) are to be evaluated with $\zeta_{2}^{2}=-\omega^{2}$. As implied by Eqs. (4.38) and (4.40), $1<b<a$. This can be proved for values or $\alpha$ and $\beta$ contained in region $R$ of Fig. 1 .

The $v$ displacement along the line $x=x_{0}$ is similarly found to be

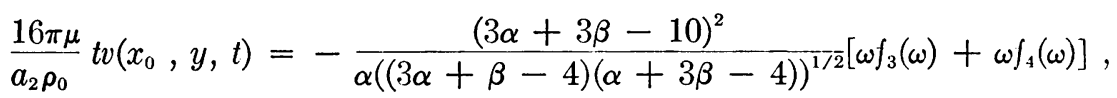

where

$$
\begin{gathered}
\beta^{-1 / 2}<\omega \leq 1, \quad f_{4}(\omega)=\frac{\alpha(2 \alpha)^{1 / 2}}{A+D+|A-D|} \\
\cdot\left[-\frac{(A+D)^{1 / 2}}{(\alpha \beta)^{1 / 2}}\left(\frac{1-\omega^{2}}{\omega^{2}-1 / \beta}\right)^{1 / 2}-(|A-D|)^{1 / 2}\right], \\
1 \leq \omega \leq b, \quad f_{3}(\omega)=-\alpha(2 \alpha)^{1 / 2} \frac{1+\frac{1}{(\alpha \beta)^{1 / 2}}\left(\frac{\omega^{2}-1}{\omega^{2}-1 / \beta}\right)^{1 / 2}}{D+D \mid)^{1 / 2}+(|A-D|)^{1 / 2}} \\
b \leq \omega \leq a, \quad f_{3}(\omega)=-\alpha(\alpha)^{1 / 2} \frac{1+\frac{1}{(\alpha \beta)^{1 / 2}}\left(\frac{\omega^{2}-1}{\omega^{2}-1 / \beta}\right)^{1 / 2}}{\left(|A|+(4 \alpha B)^{1 / 2}\right)^{1 / 2}},
\end{gathered}
$$

and

$$
a \leq \omega<\infty, \quad f_{3}(\omega)=-\alpha(2 \alpha)^{1 / 2} \frac{1+\frac{1}{(\alpha \beta)^{1 / 2}}\left(\frac{\omega^{2}-1}{\omega^{2}-1 / \beta}\right)^{1 / 2}}{(|A+D|)^{1 / 2}+(|A-D|)^{1 / 2}}
$$

It is understood that the functions $f_{3}(\omega)$ and $f_{4}(\omega)$ are identically zero for any positive $\omega$ range in which their values are not explicitly given by Eqs. (4.43)-(4.46).

It is worth noting that the expressions for the $u$ and $v$ displacements, along the line $y=y_{0}$ and $x=x_{0}$, can be written in terms of a single variable (either $\tau$ or $\omega$ ) with the exception of the multiplying time factor $t$.

5. Discussion of results. Due to the impulsive nature of the source, certain singular displacements are introduced at the wave fronts. Near these singularities the displacements, etc., become unbounded and in no sense remain small. However, the present solution can be used, by familiar methods, to obtain the displacements for other problems in which smooth distributed sources are present. In such cases the displacements would be physically meaningful (nonsingular). In this respect it is useful to remark that this very same approach is employed in isotropic elasticity theory where singular solutions are well known.

1The derivation of Eqs. (4.38) - (4.41) has tacitly assumed that $k$, the value of $\omega$ for which $A=0$, lies in the range $\beta^{-1 / 2}<k<1$. Should $k$ be larger than this (it cannot be less than $\beta^{-1 / 2}$ ), then the above expressions may differ somewhat in detail. 
Along the line $y=y_{0}$, the $u$ displacement has a reciprocal square root singularity at the wave front corresponding to $\tau=\alpha^{-1 / 2}$. The $u$ displacement is smooth at the (faster) wave front $\tau=1$. On the other hand the $v$ displacement is smooth at $\tau=\alpha^{-1 / 2}$, but has a reciprocal square root singularity at $\tau=1$.

Along the line $x=x_{0}$, the $u$ displacement has a reciprocal square root singularity at the (slower) wave front $\omega=1$ and is smooth at the wave front $\omega=\beta^{-1 / 2}$. The $v$ displacement has a reciprocal square root singularity at the wave front $\omega=\beta^{-1 / 2}$ and is finite at the front $\omega=1$. The $v$ displacement, however, is not smooth at $\omega=1$ since the left and right derivatives approach different values at this point, resulting in a kink in the curve.

As the point (in two dimensions) $x=x_{0}, y=y_{0}$ is approached

$$
\lim _{x \rightarrow x_{0}} u\left(x, y_{0}, t\right)=\lim _{v \rightarrow \nu_{0}} u\left(x_{0}, y, t\right)=u\left(x_{0}, y_{0}, t\right)=M / t,
$$

where

$$
\begin{gathered}
M=\frac{a_{1} \rho_{0}(3 \alpha+3 \beta-10)^{2}}{16 \pi \mu\left(\left(\gamma^{2}-4 \alpha \beta\right)(3 \alpha+\beta-4)(\alpha+3 \beta-4)\right)^{1 / 2}}\left[\left\{\frac{\gamma+\left(\gamma^{2}-4 \alpha \beta\right)^{1 / 2}}{2 \beta}\right\}^{1 / 2}\right. \\
\left.-\left\{\frac{\gamma-\left(\gamma^{2}-4 \alpha \beta\right)^{1 / 2}}{2 \beta}\right\}^{1 / 2}\right]\left(\beta+\left(\frac{\beta}{\alpha}\right)^{1 / 2}\right) .
\end{gathered}
$$

For the $v$ component of displacement

$$
\lim _{x \rightarrow x_{0}} v\left(x, y_{0}, t\right)=\lim _{\boldsymbol{y} \rightarrow \nu_{0}} v\left(x_{0}, y, t\right)=v\left(x_{0}, y_{0}, t\right)=N / t,
$$

where

$$
\begin{aligned}
N=\frac{a_{2} \rho_{0}(3 \alpha+3 \beta-10)^{2}}{16 \pi \mu\left(\left(\gamma^{2}-4 \alpha \beta\right)(3 \alpha+\beta-4)(\alpha+3 \beta-4)\right)^{1 / 2}}\left[\left\{\frac{\gamma+\left(\gamma^{2}-4 \alpha \beta\right)^{1 / 2}}{2 \beta}\right\}^{1 / 2}\right. & \left.-\left\{\frac{\gamma-\left(\gamma^{2}-4 \alpha \beta\right)^{1 / 2}}{2 \beta}\right\}^{1 / 2}\right]\left(1+(\alpha \beta)^{1 / 2}\right) .
\end{aligned}
$$

The first limit process in (5.1) and (5.3) corresponds to $\tau \rightarrow \infty$, while the second limit process in these equations is obtained by letting $\omega \rightarrow \infty$. Equations (5.3) and (5.4) clearly show that the displacements at the point $\left(x_{0}, y_{0}\right)$, where the impulsive source was applied, varies inversely with the time which has elapsed since the source was initiated.

Figures 3 and 4 show plots of the displacement functions, calculated from the results of Sec. 4. For these figures the values $\alpha=1 / 20, \beta=4$ were used. The corresponding values of the stretch parameters are $\lambda_{1}^{2}=3 / 43$ and $\lambda_{2}^{2}=161 / 43$. For the above $\alpha, \beta$ values, $\beta^{-1 / 2}=0.5, k=0.966, b=1.006, a=1.384$, and $\alpha^{-1 / 2}=4.472$. These curves clearly show the various singularities, kink, and limiting values discussed above.

Along the line $y=y_{0}$ the two wave fronts move with the speeds $\lambda_{1} / m$ (corresponding to the front $\tau=1$ ) and $\lambda_{1} \alpha^{1 / 2} / m$ (corresponding to the front $\tau=\alpha^{-1 / 2}$ ), while along the line $x=x_{0}$ the two wave fronts move with the speeds $\lambda_{2} \beta^{1 / 2} / m$ (corresponding to the front $\omega=\beta^{-1 / 2}$ ) and $\lambda_{2} / m$ (corresponding to the front $\omega=1$ ). The fact that the wave fronts travel with different speeds in different directions is, of course, caused by the anisotropic nature of the equations of motion. 


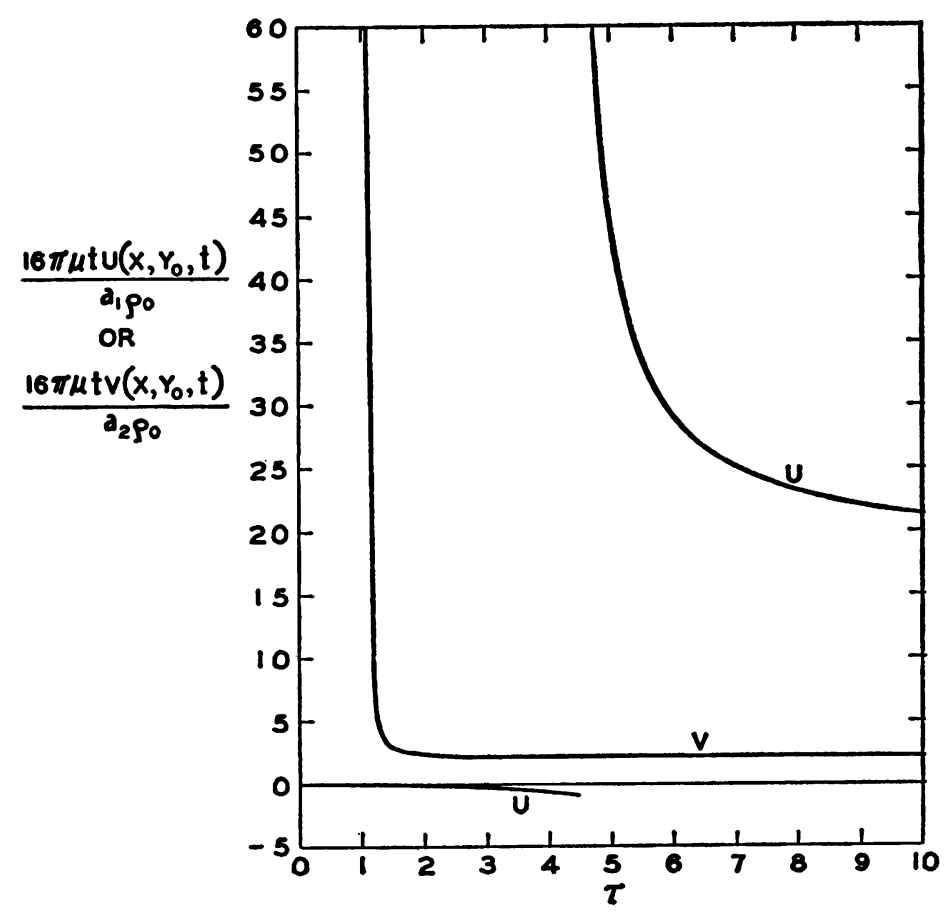

FIG. 3. Variation of $u$ and $v$ displacement components with $\tau$ along the $y=y_{0}$. Time is fixed and $\alpha=$ $0.05, \beta=4$.

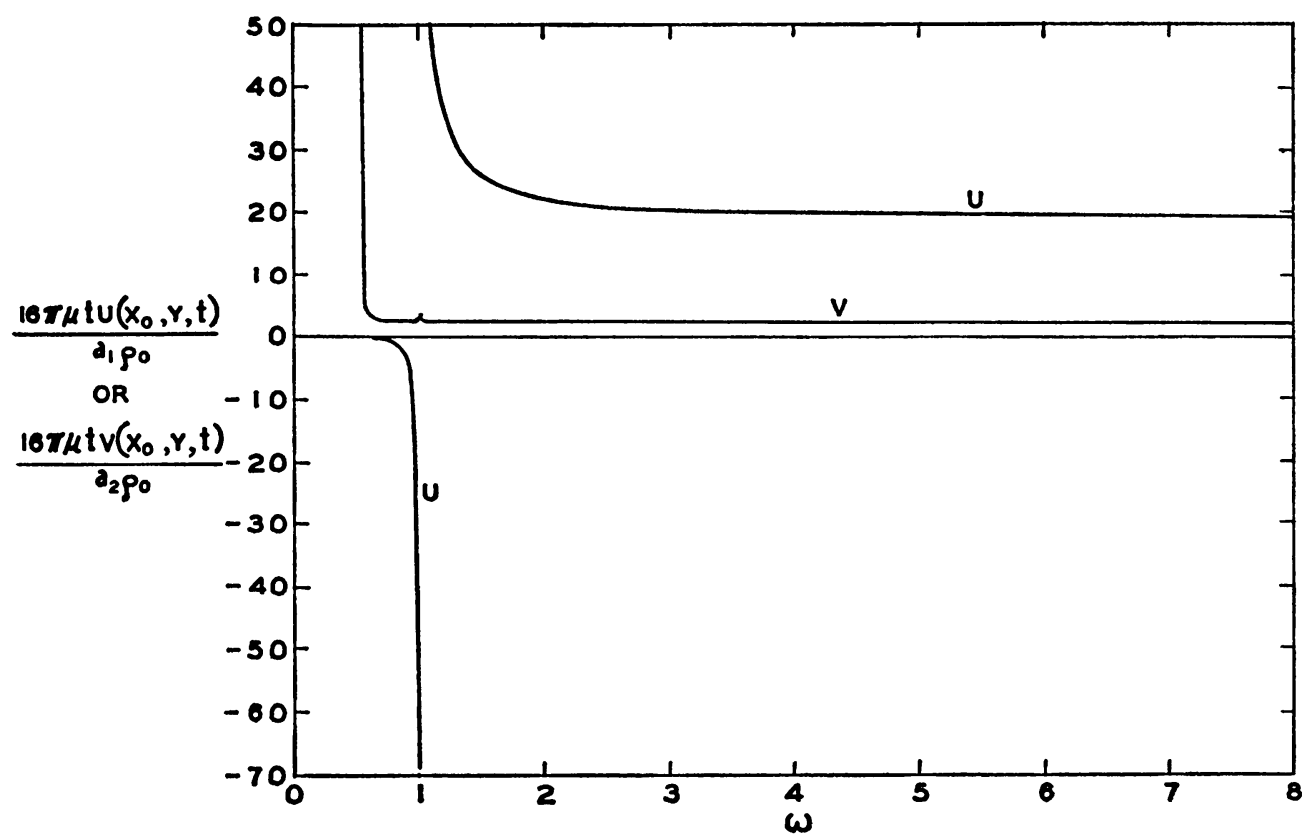

Fig. 4. Variation of $u$ and $v$ displacement components with $\omega$ along the line $x=x_{0}$. Time is fixed and $\alpha=0.05, \beta=4$. 


\section{ReFERENCES}

1. R. A. Scott and J. Miklowitz, Office of Naval Research, Project No. NR-064-487, Technical Report No. 4 (1966)

2. N. Cameron and G. Eason, Quart. J. Mech. Appl. Math. 20, 23 (1967)

3. R. Courant and D. Hilbert, Methods of mathematical physics, Vol. II, Wiley, New York, 1962

4. L. Cagniard, Reflection and refraction of progressive seismic waves, McGraw-Hill, New York, 1962

5. E. A. Kraut, Rev. Geophys. 1, 401 (1963)

6. M. J. Lighthill, Philos. Trans. Roy. Soc. A 252, 397 (1960)

7. F. John, Plane waves and spherical means applied to partial differential equations, Wiley, New York, 1955

8. I. M. Gelfand and G. E. Shilov, Generalized functions, Vol. I, Academic Press, New York, 1964 